\title{
Ceguera secundaria a neuropatía óptica isquémica por una disección carotídea espontánea. Caso clínico
}

Marcelo Miranda C, Pablo Venegas F, Marianne Kagi G. Acute ischemic optic neuropathy
causing permanent blindness as a
presentation of a carotid dissection.
Report of one case

In young and middle age subjects, spontaneous carotid dissection is an increasingly recognized cause of ischemic stroke. Their usual presentation is facial pain with a Horner syndrome and a contra lateral paresis. Blindness has been reported as a presenting symptom in only a few cases. We report a 50 years old man who presented with amblyopia in the left eye, without periocular pain. Fundoscopy showed papilledema and a peripapillar hemorrhage, compatible with an ischemic optic neuropathy. A magnetic resonance angiography confirmed a left carotid dissection (Rev Méd Chile 2003; 131: 1042-44).

(Key Words: Blindness; Carotid artery diseases; Optic neuropathy, ischemic)

Recibido el 28 de marzo, 2003. Aceptado en versión corregida el 11 de julio, 2003.

Departamento de Neurología, Hospital Clínico de la Universidad de Chile. Santiago, Chile.

L a disección carotídea espontánea es una causa cada vez más reconocida de accidente vascular isquémico, siendo la causa en 10 a $25 \%$ de los casos de presentación en la edad de adulto joven ${ }^{1}$. Afecta todos los grupos etáreos, incluyendo niños, pero existe una mayor incidencia en la quinta década de la vida ${ }^{1}$. La presentación habitual es la presencia de dolor cervical o facial, asociado a un síndrome de Horner ipsilateral; es decir miosis y ptosis palpebral, asociado a déficit motor y/o sensitivo contralateral. Es infrecuente el compromiso de otros pares craneales destacando

Correspondencia a: Marcelo Miranda C. Llewellyn Jones 1530, Santiago. E mail: marcelomiranda@terra.cl el compromiso del duodécimo par o hipogloso. Aún más inusual, no existen más de 8 casos descritos en la literatura ${ }^{2-5}$, es la presentación como amaurosis secundaria a neuropatía óptica isquémica (NOI) o por infarto de la arteria central retina debido a embolización a arteria oftálmica proveniente de la disección carotídea. Presentamos un paciente que debuta con un cuadro agudo de ceguera monocular y cuyo estudio confirma la presencia de una disección carotídea ipsilateral.

\section{CAso Cúnico}

Paciente de 50 años, sin antecedentes mórbidos, quién en actividad refiere la aparición de déficit visual brusco y progresivo a la ceguera en 3 a $4 \mathrm{~h}$ 
en ojo izquierdo, sin dolor periocular, acompañado de dolor cervical de leve intensidad con irradiación occipital y frontal. Consultó en urgencia oftalmológica donde se diagnosticó neuritis óptica. En examen neurológico destacaba visión bulto en su ojo izquierdo, defecto pupilar aferente, con edema papilar con hemorragia peripapilar (Figura 1), sin otras alteraciones retinales lo que era compatible con una neuropatía óptica isquémica; no se observó defecto motor ni signos piramidales. Fue enviado a hospitalizar en 2 ocasiones siendo rechazado de servicios de urgencia. Resonancia Magnética Cerebral con Angio Resonancia de vasos cervicales confirmó la presencia de disección carotídea izquierda (Figura 2), en el intertanto desarrolló hemiparesia derecha que revirtió en 2-3 días. Fue tratado con heparina, sin presentar nuevos fenómenos isquémicos, pero el severo defecto visual queda como secuela.

\section{Discusión}

Nuestra observación señala la relevancia de considerar la posibilidad diagnóstica de disección carotídea en un paciente que se presenta con pérdida aguda de la visión como principal manifestación clínica. La importancia radica en la necesidad de un diagnóstico precoz y el inicio de tratamiento anticoagulante que puede evitar la aparición de un nuevo daño neurológico, si bien no revierte el daño ya establecido. A pesar de no existir estudios controlados que avalen la utilidad de la anticoagulación, la formación de un trombo local en la pared vascular dañada y el riesgo de embolias arterio-arteriales (que puede explicar en este caso la presencia de neuropatía óptica isquémica), hacen recomendable su uso ${ }^{1}$.

En el enfermo presentado se planteó en urgencia el diagnóstico de neuritis óptica: este es un cuadro en general inflamatorio y desmielinizante que provoca disminución de visión de aparición no tan aguda como en nuestro paciente, acompañado de dolor a la movilización ocular y evidentemente no está acompañado de las complicaciones que presentó el paciente ${ }^{6}$. El diagnóstico de disección 
carotídea puede ser difícil de realizar si no se busca dirigidamente en especial en los pacientes que se presentan sin dolor cervical o facial asociado ${ }^{2}$.

Las disecciones de vasos arteriales cervicales constituyen sólo $2 \%$ de las causas de accidente vascular pero alcanzan $20 \%$ en pacientes jóvenes, pudiendo ser espontáneas o secundarias a un traumatismo que puede ser mínimo (hiperextensiones del cuello, maniobras quiroprácticas) ${ }^{1}$. La disección arterial puede ocurrir en cualquier arteria del organismo pero es más frecuente en los segmentos extracraneales de las arterias carótida y vertebral que están expuestos a una mayor movilidad. Se produce una solución de continuidad de la íntima del vaso que permite el paso de la sangre a través de la pared arterial formando un hematoma intramural. Este hematoma está ubicado en la túnica media arterial y puede llevar a la estenosis del vaso o a una formación aneurismáti$\mathrm{ca}^{1}$. La confirmación diagnóstica se hace en base al estudio radiológico en que los métodos de elección son la angio-resonancia de los vasos cervicales y si no es confirmatoria se debe recurrir a la angiografía convencional ${ }^{1}$.

La neuropatía óptica isquémica (NOI) es un infarto de la parte anterior, o menos frecuentemente la parte posterior (retrobulbar) del nervio óptico. Es debido a una disminución del flujo sanguíneo en las pequeñas arterias que lo nutren, derivadas de la arteria central de la retina y la arteria oftálmica que son ramas terminales de la arteria carótida interna. Se caracteriza clínicamente por una pérdida súbita, generalmente indolora de la visión monocular, con defecto pupilar aferente y un déficit visual monocular ${ }^{5}$. El fondo de ojo

\section{REFERENCIAS}

1. Schievink W. Spontaneus Dissection of the Carotid and Vertebral Arteries. N Engl Med 2001: 344: 898906.

2. Lee SK, Kwon SU, Ahn H, KIm J. Acute isolated monocular blindness and painless carotid dissection. Neurology 1999; 53: 1155.

3. Newman N, Kune L, Leifer D, Lersell S. Ocular Stroke and Carotid Dissection. Neurology 1989; 39: 1462-4. revela una papila hiperémica con hemorragias peripapilares cuando se afecta la parte anterior del nervio (neuropatía óptica isquémica anterior) como en nuestro caso, pero es normal si se afecta la parte retrobulbar. No se observan cambios retinales como edema o la presencia de una mácula rojo-cereza. En la etiología de la NOI se describe una forma arterítica, generalmente por arteritis de células gigantes (se observan en personas adultas mayores, asociado a importante dolor y una elevada velocidad de sedimentación). La forma no arterítica rara vez se asocia a dolor y se debe a aterotrombosis de los pequeños vasos 0 como consecuencia de hipotensión que afectan la circulación del nervio óptico que es de tipo limítrofe o más raramente por una enfermedad carotídea como la disección arterial. El mecanismo de la isquemia cuando la NOI se debe a disección arterial se discute si es por un fenómeno hemodinámico, por disminución de flujo o por embolias arterio-arteriales $^{5}$. La presencia de dolor en un paciente en la edad media afectado de un cuadro compatible con NOI debe hacer sospechar la presencia de una disección carotídea ${ }^{5}$.

Esta comunicación enfatiza la necesidad de considerar el diagnóstico de disección carotídea en pacientes que se presentan con pérdida aguda de la visión monocular, aun cuando no existan signos neurológicos asociados ni historia clara de trauma cervical o dolor cervical. La posibilidad además de revertir el déficit neurológico isquémico en la actualidad con el uso de trombolíticos, que ya se han usado con éxito en casos de disecciones arteriales, hace necesario considerar este diagnóstico.

4. TSAI RK, Sun CY. Spontaneous Carotid Dissection presenting as Ischaemic Optic Neuropathy. B J Ophthalmol 1997; 81: 513.

5. Biousse V, Schaison M, Toubul PJ, D’AnglejanChatiLon J, Bousser MG. Ischemic Optic Neuropathy associated with internal carotid dissection. Arch Neurol 1998; 55: 715-9.

6. Alvarez G. Neuritis óptica en: Arriagada C, Nogales J. Eds. Esclerosis Múltiple. Una visión Ibero-Panamericana. Santiago Eds. ArryNog 2002; 333-44. 